Pacific

Journal of

Mathematics

THE KAUFFMAN BRACKET SKEIN MODULE OF SURGERY ON A $(2,2 b)$ TORUS LINK

JOHN M. HARRIS

Volume $245 \quad$ No. 1

March 2010 


\title{
THE KAUFFMAN BRACKET SKEIN MODULE OF SURGERY ON A $(2,2 b)$ TORUS LINK
}

\author{
JOHN M. HARRIS
}

\begin{abstract}
We show that the Kauffman bracket skein modules of certain manifolds obtained from integral surgery on a $(2,2 b)$ torus link are finitely generated, and list the generators for select examples.
\end{abstract}

\section{Introduction}

Kauffman [1988] presented an elegant construction of the Jones polynomial, an invariant of oriented links in $S^{3}$, by constructing a new invariant, the Kauffman bracket polynomial. The Kauffman bracket is an invariant of unoriented framed links in $S^{3}$, defined by the skein relations

(1)

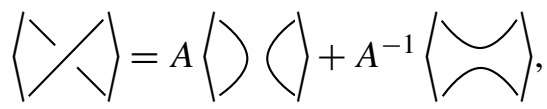

(2) $\langle L \cup$ unknot $\rangle=\left(-A^{-2}-A^{2}\right)\langle L\rangle$.

For the invariant to be well defined, one also must normalize it by choosing a value for the empty link, for instance $\langle$ empty link $\rangle=1$.

Alternatively, we can use the skein relations to construct a module of equivalence classes of links in $S^{3}$, or, for that matter, in any oriented 3-manifold. See Przytycki [1991] and Turaev [1988].

Definition 1. Let $N$ be an oriented 3-manifold, and let $R$ be a commutative ring with identity, with a specified unit $A$. The Kauffman bracket skein module of $N$, denoted $S(N ; R, A)$, or simply $S(N)$, is the free $R$-module generated by the framed isotopy classes of unoriented links in $N$, including the empty link, quotiented by the skein relations that define the Kauffman bracket.

Since every crossing and unknot can be eliminated from a link in $S^{3}$ by the skein relations, $S\left(S^{3}\right)$ is generated by the empty link. Kauffman's argument that his bracket polynomial is well defined shows that $S\left(S^{3}\right)$ is free on the empty link.

For $R=\mathbb{Z}\left[A^{ \pm 1}\right]$, Hoste and Przytycki have computed the skein modules of all of the closed, oriented manifolds of genus 1: In [1993], they computed $S(L(p, q))$,

MSC2000: 57M27.

Keywords: skein theory, Poincaré homology sphere, quaternionic manifold. 
$1=(O=O)$

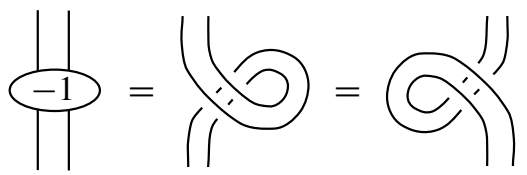

Figure 1. Examples of twist notation.

which is free on $\lfloor p / 2\rfloor+1$ generators, and in [1995] they computed $S\left(S^{1} \times S^{2}\right) \cong$ $\mathbb{Z}\left[A^{ \pm 1}\right] \oplus\left(\bigoplus_{i=1}^{\infty} \mathbb{Z}\left[A^{ \pm 1}\right] /\left(1-A^{2 i+4}\right)\right)$. Over $\mathbb{Z}\left[A^{ \pm 1}\right]$, localized by inverting all of the cyclotomic polynomials, Gilmer and the author have computed the skein module of the quaternionic manifold [Gilmer and Harris 2007].

Additionally, Bullock [1997a] has determined whether or not the skein module obtained from integral surgery on a trefoil is finitely generated. In this paper, we obtain a similar result for integral surgery on a $(2,2 b)$ torus link.

Notation 2. For any integer $n$, let

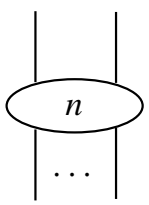

denote $n$ full twists in the depicted strands. For example, see Figure 1.

Definition 3. We define $M(\alpha, \beta, \gamma)$ to be the manifold obtained by surgery on the torus link

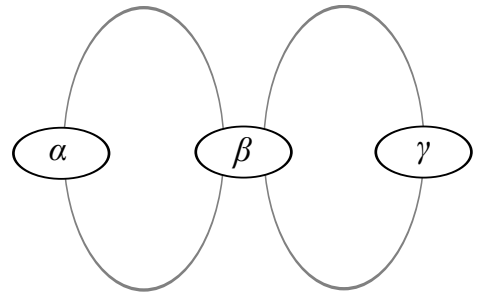

with the blackboard framing.

Theorem 4. For all integers $\alpha, \beta$, and $\gamma$ such that

$$
\begin{aligned}
a & =|\alpha|>1, & b & =|\beta|>1, & c & =|\gamma|>1, \\
a^{-1} & <b^{-1}+c^{-1}, & b^{-1} & <a^{-1}+c^{-1}, & c^{-1} & <a^{-1}+b^{-1},
\end{aligned}
$$

$S(M(\alpha, \beta, \gamma))$ is finitely generated.

For specific values of $\alpha, \beta$, and $\gamma$, we can use brute-force computation to refine our result, explicitly listing generating sets for $S(M(\alpha, \beta, \gamma))$. 
Notation 5. We refer to the collection of loops

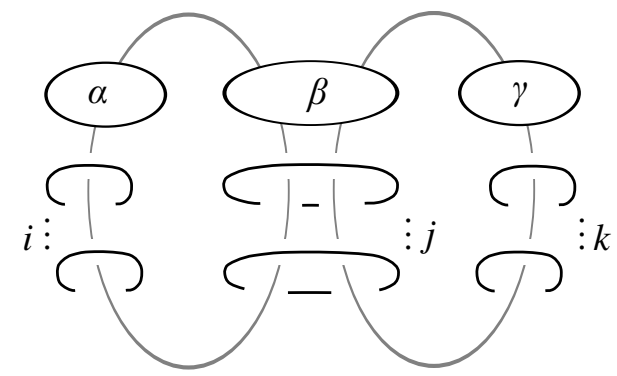

in $M(\alpha, \beta, \gamma)$ using the algebraic notation $x^{i} y^{j} z^{k}$.

In particular, we obtain the following for $S(M(2,-2,2)), S(M(3,-2,3))$, and $S(M(3,-2,5))$, which are respectively the skein modules of the 3-, 4-, and 5-fold branched cyclic coverings of $S^{3}$ over the trefoil, as listed by Rolfsen [1976].

\begin{tabular}{ccccc}
\hline$\alpha$ & $\beta$ & $\gamma$ & fundamental group & generators \\
\hline 2 & -2 & 2 & quaternion group & $1, z, z^{2}, y, x$ \\
3 & -2 & 3 & binary tetrahedral group & $1, z, z^{2}, z^{3}, y, x, x^{2}$ \\
3 & -2 & 5 & binary icosahedral group & $1, z, z^{2}, z^{3}, z^{4}, z^{5}, y, x, x^{2}$ \\
\hline
\end{tabular}

Note that the generating set for the skein module of the quaternionic manifold essentially coincides with what was shown in [Gilmer and Harris 2007] over the ring $R^{\prime}$ obtained from $\mathbb{Z}\left[A^{ \pm 1}\right]$ by inverting the multiplicative set generated by the elements of the set $\left\{A^{n}-1 \mid n \in \mathbb{Z}^{+}\right\}$. Since any dependence relation over $\mathbb{Z}\left[A^{ \pm 1}\right]$ would hold over $R^{\prime}$ and since $S\left(M(2,-2,2) ; R^{\prime}, A\right)$ is a free module of rank 5 , we obtain the following:

Corollary 6. $S\left(M(2,-2,2) ; \mathbb{Z}\left[A^{ \pm 1}\right], A\right)$ is a free module of rank 5.

This result was conjectured in [Gilmer and Harris 2007]. The quaternionic manifold is the first closed, irreducible, genus two 3-manifold whose Kauffman bracket skein module has been computed.

\section{Twists and loops}

Twists have many useful properties, a few of which are listed in Figure 2. Note that, to obtain clearer diagrams, we represent a fixed but arbitrary number of parallel strands with a thick line.

We are most interested in using skein relations and isotopy to rewrite one strand, twisted with others, as a linear combination involving loops encircling the others, as in Figure 3.

In fact, by repeating the steps performed in Figure 3, we obtain this: 
122

JOHN M. HARRIS

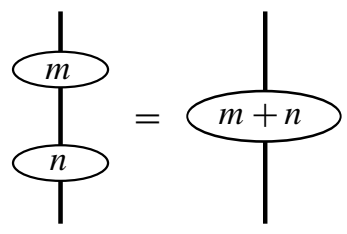

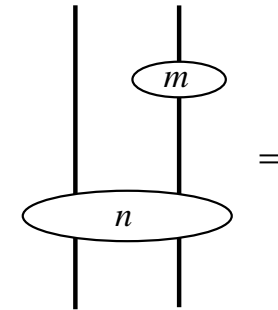

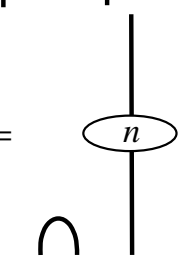

Figure 2. Useful properties of twists.

Lemma 7. For each integer $n>0$,

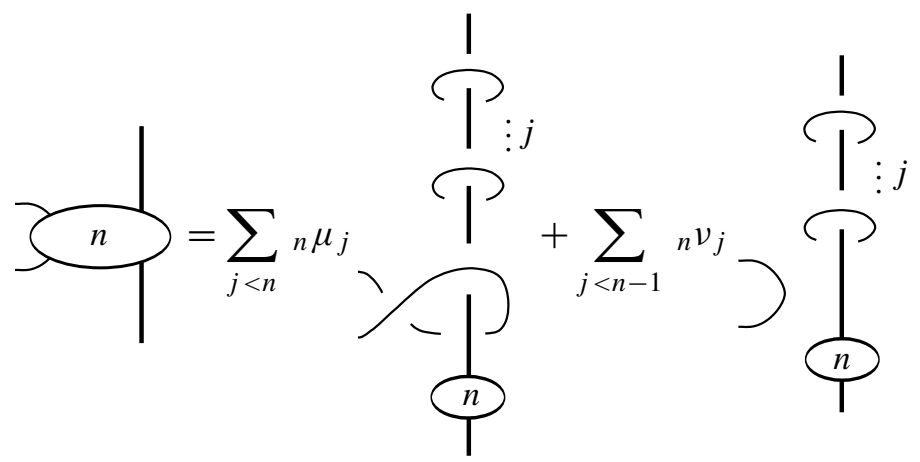

for some ${ }_{n} \mu_{0}, \ldots,{ }_{n} \mu_{n-1},{ }_{n} \nu_{0}, \ldots,{ }_{n} v_{n-2} \in R$ with ${ }_{n} \mu_{n-1}=A^{n-1}$, and

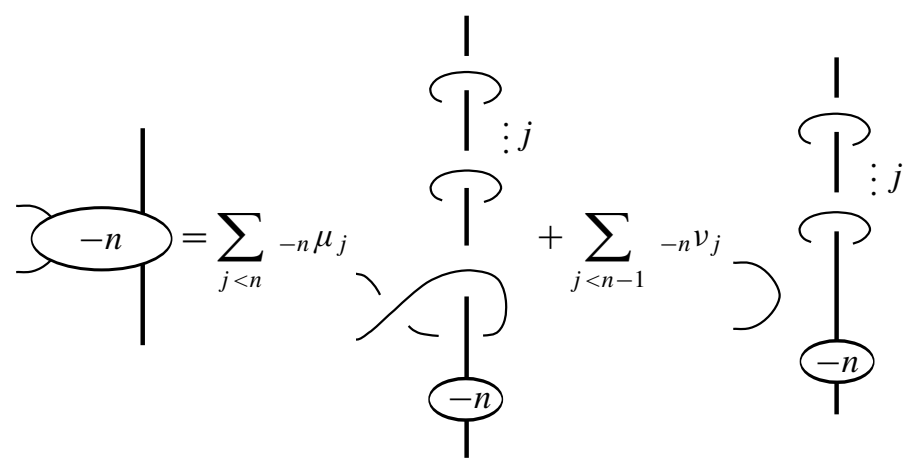

for some ${ }_{-n} \mu_{0}, \ldots,{ }_{-n} \mu_{n-1},{ }_{-n} \nu_{0}, \ldots,{ }_{n} \nu_{n-2} \in R$ with ${ }_{-n} \mu_{n-1}=A^{1-n}$.

Proof. For $n=1$ and $n=2$, the result is obtained in Figure 3. 


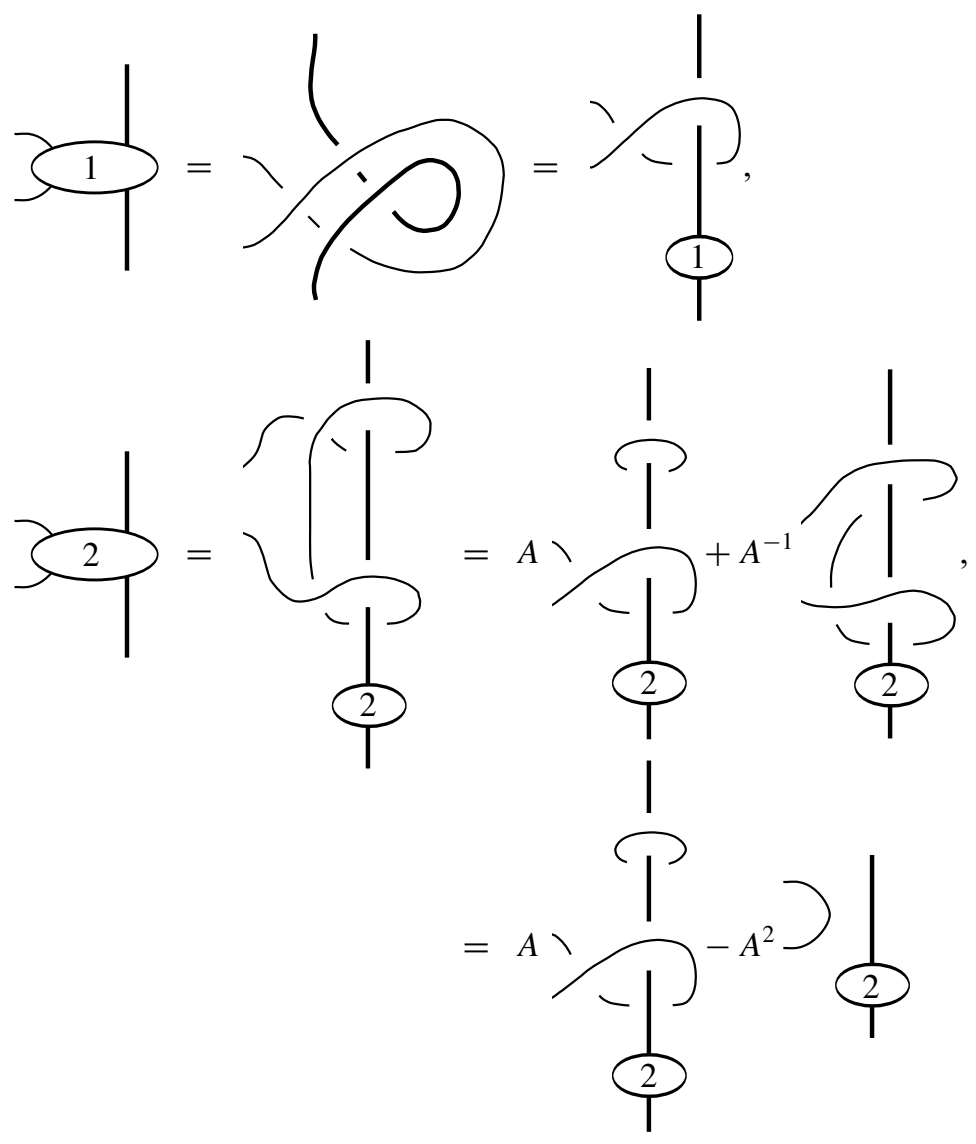

Figure 3. Examples of rewriting twists.

Let $n>2$, and suppose that the result holds for all $k<n$. Then

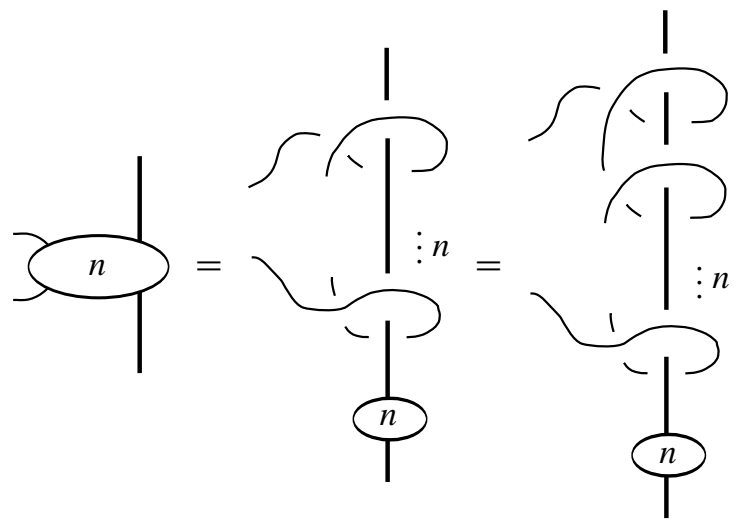



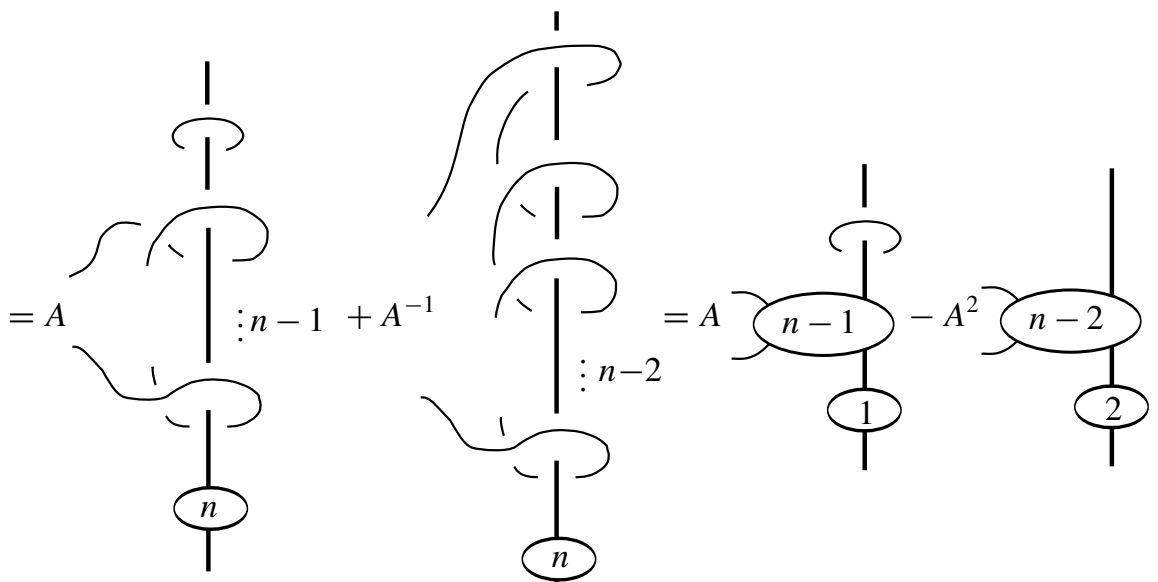

Hence, the first equation follows by induction on $n$. The second equation can be obtained by reversing all of the crossings in the first.

By rotating the diagrams in Lemma 7 by 180 degrees, we obtain another:

Lemma 8. For each integer $n>0$,

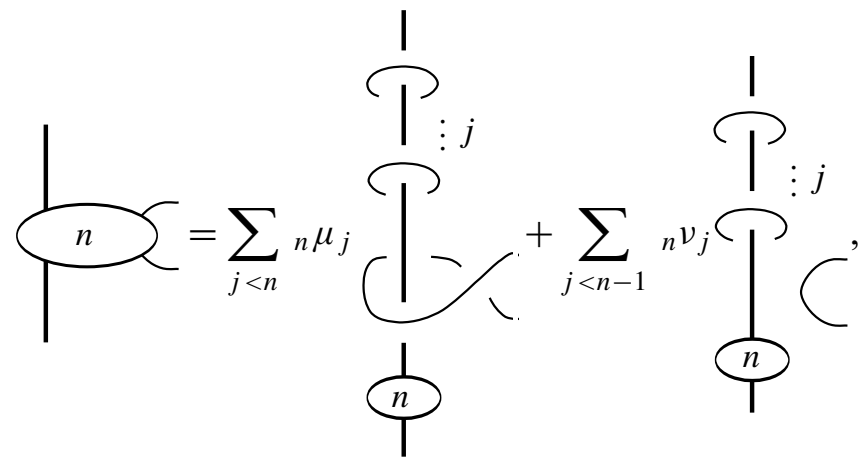

where ${ }_{n} \mu_{n-1}=A^{n-1}$, and

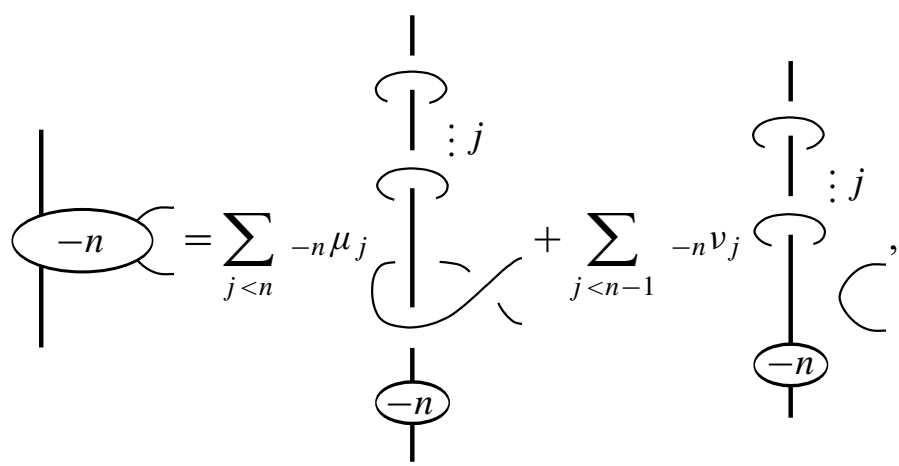

where ${ }_{-n} \mu_{n-1}=A^{1-n}$. 
In particular, if a component of a link is only twisted about one set of other strands, we obtain an immediate corollary of Lemma 7:

Lemma 9. For each integer $n>0$,

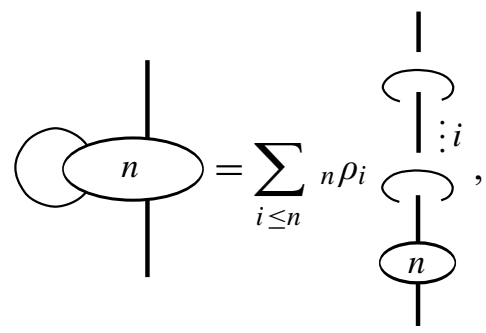

for some ${ }_{n} \rho_{0}, \ldots,{ }_{n} \rho_{n} \in R$ with ${ }_{n} \rho_{n}=-A^{n+2}$, and

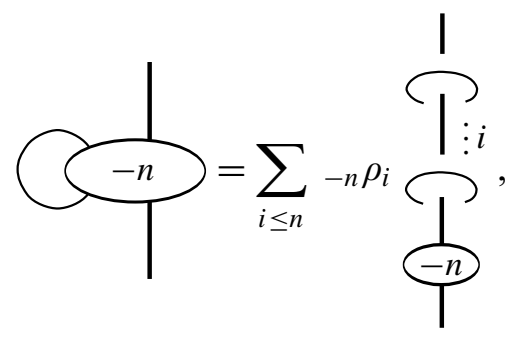

for some ${ }_{-n} \rho_{0}, \ldots,{ }_{-n} \rho_{n} \in R$ with ${ }_{-n} \rho_{n}=-A^{-n-2}$.

Similarly, the corollary of Lemma 8:

Lemma 10. For each integer $n>0$,

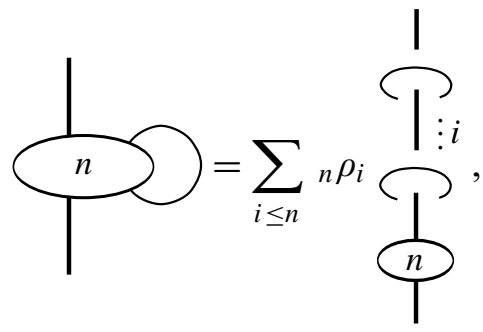

where ${ }_{n} \rho_{n}=-A^{n+2}$, and

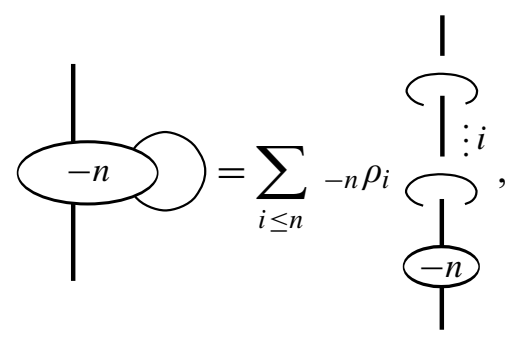

where $_{-n} \rho_{n}=-A^{-n-2}$. 
Remark 11. We only need the explicitly computed coefficients in Lemmas 7-10 for the proofs that follow, but the other coefficients are not too difficult to compute explicitly as well: For $n>0$, we have ${ }_{n} \rho_{j}=-A^{3}{ }_{n} \mu_{j-1}+\left(-A^{-2}-A^{2}\right)_{n} v_{j}$, and for $n>2$, we have ${ }_{n} \mu_{j}=A_{n-1} \mu_{j-1}-A^{2}{ }_{n-2} \mu_{j}$ and ${ }_{n} v_{j}=A_{n-1} v_{j-1}-A^{2}{ }_{n-2} v_{j}$, yielding

$$
{ }_{n} \mu_{j}= \begin{cases}(-1)^{(n-j-1) / 2}\left(\begin{array}{c}
(n+j-1) / 2 \\
j
\end{array}\right) A^{n-1} & \text { for } n+j \text { odd and } 0 \leq j<n \\
0 & \text { otherwise }\end{cases}
$$

and

$$
{ }_{n} v_{j}= \begin{cases}(-1)^{(n-j) / 2}\left(\begin{array}{c}
(n+j-2) / 2 \\
j
\end{array}\right) A^{n} & \text { for } n+j \text { even and } 0 \leq j<n-1, \\
0 & \text { otherwise. }\end{cases}
$$

Suppose that a component of a link is twisted with two sets of strands. While more complicated than in the cases previously considered, it is still possible to rewrite the component as a linear combination of loops around the other strands:

Lemma 12. For all integers $m, n>0$,
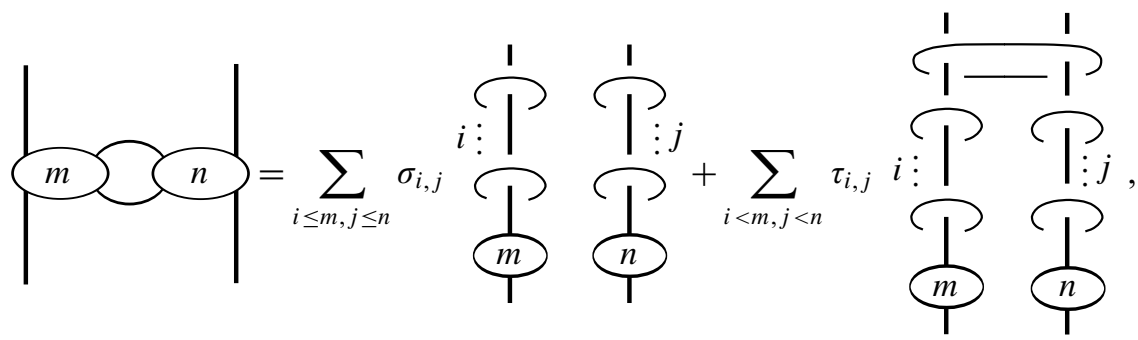

for some $\sigma_{0,0}, \ldots, \sigma_{m, n}, \tau_{0,0}, \ldots, \tau_{m-1, n-1} \in R$ with $\sigma_{m, n}=-A^{m+n+2}$.

Proof. Applying Lemma 7, and then applying Lemma 8 to each diagram of the resulting linear combination, we have

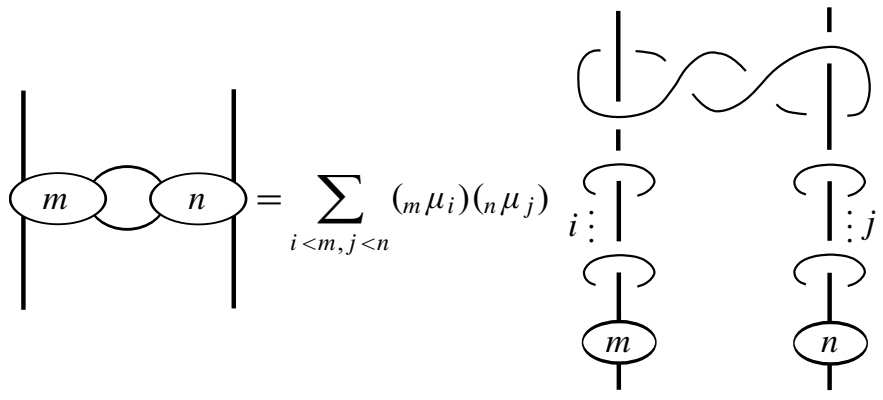




$$
+\sum_{i \leq m, j<n-1}-A^{3}\left({ }_{m} \mu_{i}\right)\left({ }_{n} v_{j}\right)
$$

Since

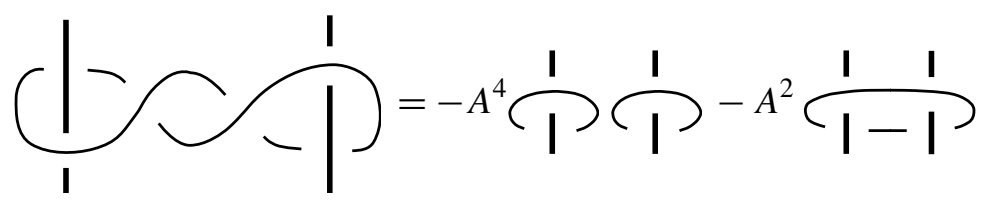

the result follows.

Lemma 13. For all integers $m, n>0$,

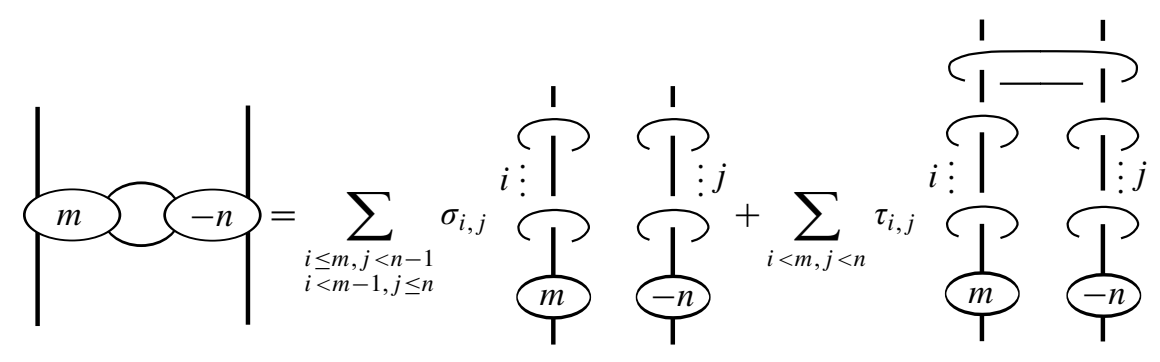

where $\tau_{m-1, n-1}=A^{m-n}$. 
Proof. Applying Lemma 7, and then applying Lemma 8 to each diagram of the resulting linear combination, we get

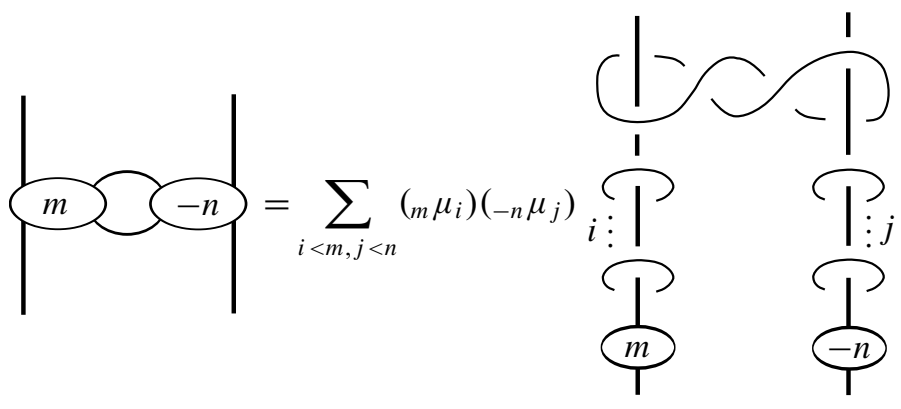

$$
\left.\left.+\sum_{i<m-1, j \leq n}-A^{-3}\left(m_{m} v_{i}\right)\left({ }_{-n} \mu_{j}\right) A_{i<m, j<n-1}^{3}-A_{m} \mu_{i}\right)_{-n} v_{j}\right)
$$

By an argument similar to that for Lemma 13, we obtain this:

Lemma 14. For all integers $m, n>0$,

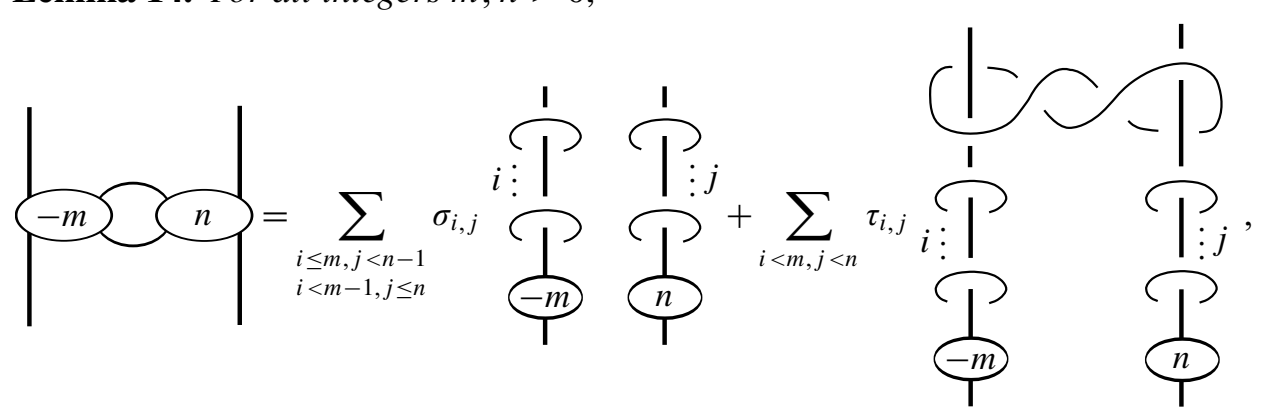

where $\tau_{m-1, n-1}=A^{n-m}$. 
By an argument similar to that for Lemma 12, we obtain this:

Lemma 15. For all integers $m, n>0$,
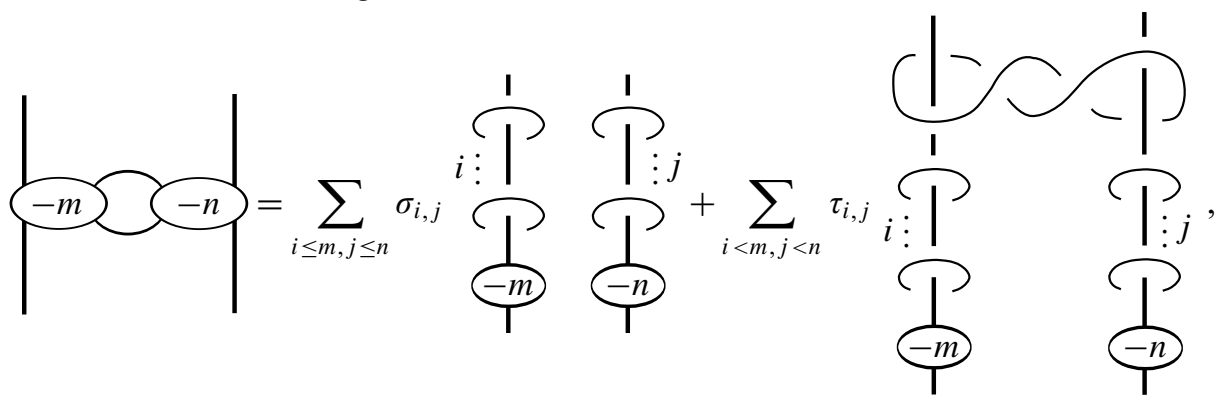

where $\sigma_{m, n}=-A^{-m-n-2}$.

Remark 16. In Lemmas $12-15$, the coefficients depend on the number of twists as in Lemmas 7-10: for example, $\sigma_{i, j}$ would be written more precisely as ${ }_{m, n} \sigma_{i, j}$ in Lemma 12. Since we do not need to refer to the coefficients by name in the following sections, we have simplified the notation for the sake of readability.

\section{Finitely generating the skein module}

Since all links in the exterior of the surgery description of $M(\alpha, \beta, \gamma)$ can be isotoped into a genus two handlebody and since the skein relations allow us to remove all crossings in a diagram, $S(M(\alpha, \beta, \gamma))$ is generated by $\left\{x^{i} y^{j} z^{k}\right\}$.

Definition 17. For $a=|\alpha|, b=|\beta|, c=|\gamma|>0$, we define a strict linear ordering on the generating set $\left\{x^{i} y^{j} z^{k}\right\}$ of $M(\alpha, \beta, \gamma)$. We say $x^{i} y^{j} z^{k}<x^{m} y^{n} z^{p}$ if any of the following hold:

- $\frac{i}{a}+\frac{j}{b}+\frac{k}{c}<\frac{m}{a}+\frac{n}{b}+\frac{p}{c}$.

- $\frac{i}{a}+\frac{j}{b}+\frac{k}{c}=\frac{m}{a}+\frac{n}{b}+\frac{p}{c}, \quad i(k+1)<m(p+1)$.

- $\frac{i}{a}+\frac{j}{b}+\frac{k}{c}=\frac{m}{a}+\frac{n}{b}+\frac{p}{c}, \quad i(k+1)=m(p+1)$,

$$
\max \left(\frac{j}{b}, \frac{k}{c}\right)<\max \left(\frac{n}{b}, \frac{p}{c}\right) .
$$

- $\frac{i}{a}+\frac{j}{b}+\frac{k}{c}=\frac{m}{a}+\frac{n}{b}+\frac{p}{c}, \quad i(k+1)=m(p+1)$,

$$
\max \left(\frac{j}{b}, \frac{k}{c}\right)=\max \left(\frac{n}{b}, \frac{p}{c}\right), \quad j<n .
$$

- $\frac{i}{a}+\frac{j}{b}+\frac{k}{c}=\frac{m}{a}+\frac{n}{b}+\frac{p}{c}, \quad i(k+1)=m(p+1)$,

$$
\max \left(\frac{j}{b}, \frac{k}{c}\right)=\max \left(\frac{n}{b}, \frac{p}{c}\right), \quad j=n, \quad k<p .
$$


Suppose that

$$
a, b, c>1, \quad a^{-1}<b^{-1}+c^{-1}, \quad b^{-1}<a^{-1}+c^{-1}, \quad c^{-1}<a^{-1}+b^{-1} .
$$

By sliding over an attached 2-handle, we obtain useful relations:

Definition 18. The Type I relation is

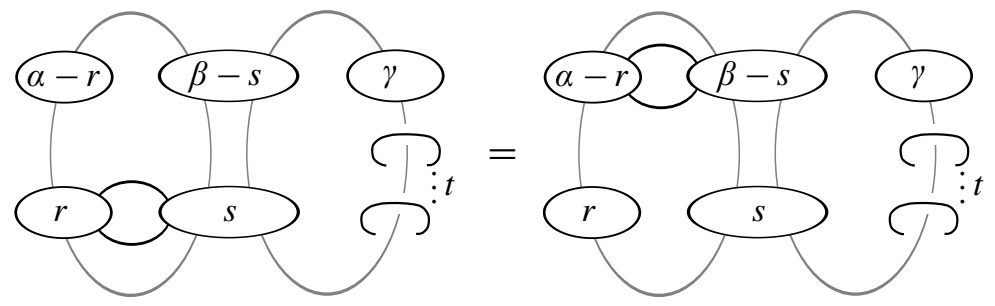

First, note that by Lemmas $12-15$, each side of the relation can be written as a linear combination of loops of the form $x^{i} y^{j} z^{k}$, since for all nonnegative integers $u, v$, and $w$,

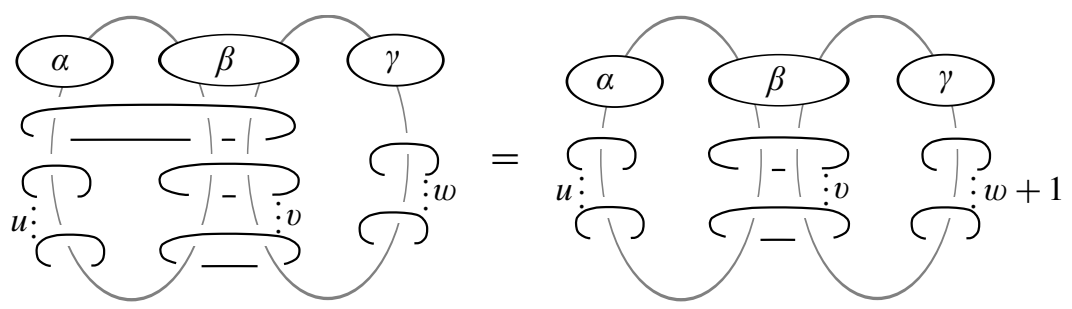

Note that when $r \geq 0$ and $s \geq 0$, the greatest term appearing on the left side of the Type I relation, rewritten as a linear combination of loops, is $x^{r} y^{s} z^{t}$ :

When $r, s>0$, by Lemma 12, $x^{r} y^{s} z^{t}$ and $x^{r-1} y^{s-1} z^{t+1}$ appear as the greatest terms of their respective types.

Since $c^{-1}<a^{-1}+b^{-1}$,

$$
\frac{r}{a}+\frac{s}{b}+\frac{t}{c}>\left(\frac{r}{a}+\frac{s}{b}+\frac{t}{c}\right)+\left(-\frac{1}{a}-\frac{1}{b}+\frac{1}{c}\right)=\frac{r-1}{a}+\frac{s-1}{b}+\frac{t+1}{c} .
$$

When either $r=0$ or $s=0$, the claim follows by Lemma 9 or Lemma 10. When both are 0 , the claim follows trivially.

Also note that as long as $r>0$ or $s>0$, the leading coefficient is $-A^{r+s+2}$.

Similarly, when $r \leq 0$ and $s \leq 0$, the greatest term appearing on the left side of the Type I relation is $x^{-r} y^{-s} z^{t}$, and as long as both are nonzero, its coefficient is $-A^{r+s-2}$.

When $r>0$ and $s<0$, the greatest term appearing on the left side of the Type I relation is $x^{r-1} y^{-s-1} z^{t+1}$ : 
By Lemma 13, $x^{r-1} y^{-s-1} z^{t+1}, x^{r-2} y^{-s} z^{t}$, and $x^{r} y^{-s-2} z^{t}$ appear as the greatest terms of their respective types. Since $b^{-1}<a^{-1}+c^{-1}$,

$\frac{r-1}{a}+\frac{-s-1}{b}+\frac{t+1}{c}>\left(\frac{r-1}{a}+\frac{-s-1}{b}+\frac{t+1}{c}\right)+\left(-\frac{1}{a}+\frac{1}{b}-\frac{1}{c}\right)=\frac{r-2}{a}+\frac{-s}{b}+\frac{t}{c}$.

Since $a^{-1}<b^{-1}+c^{-1}$,

$\frac{r-1}{a}+\frac{-s-1}{b}+\frac{t+1}{c}>\left(\frac{r-1}{a}+\frac{-s-1}{b}+\frac{t+1}{c}\right)+\left(\frac{1}{a}-\frac{1}{b}-\frac{1}{c}\right)=\frac{r}{a}+\frac{-s-2}{b}+\frac{t}{c}$.

Also note that in this case, the leading coefficient is $A^{r+s}$.

Similarly, when $r<0$ and $s>0$, the greatest term appearing on the left side is $x^{-r-1} y^{s-1} z^{t+1}$, with coefficient $A^{r+s}$.

Likewise, the greatest term on the right side is $x^{|\alpha-r|-1} y^{|\beta-s|-1} z^{t+1}$, when $\alpha-r$ and $\beta-s$ are nonzero with different signs, and the greatest term on the right side is $x^{|\alpha-r|} y^{|\beta-s|} z^{t}$ otherwise.

By sliding over the other attached 2-handle, we obtain additional relations:

Definition 19. The Type II relation is

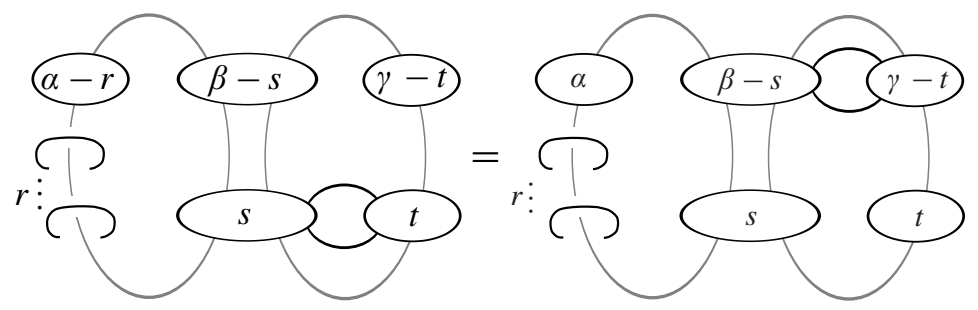

As with the Type I relation, each side of the relation can be rewritten as a linear combination of loops of the form $x^{i} y^{j} z^{k}$.

Also, as with the Type I relation, the greatest term appearing on the left side of the Type II relation is $x^{r+1} y^{|s|-1} z^{|t|-1}$ when the signs of $s$ and $t$ differ, with coefficient $A^{s+t}$. Otherwise, the greatest term appearing on the left side is $x^{r} y^{|s|} z^{|t|}$, and as long as one of $s$ and $t$ are nonzero, the leading coefficient is $-A^{s+t \pm 2}$.

Finally, as with the Type I relation, the greatest term on the right side of the Type II relation is $x^{r+1} y^{|\beta-s|-1} z^{|\gamma-t|-1}$ when the signs of $\beta-s$ and $\gamma-t$ differ, and the greatest term appearing on the left side is $x^{r} y^{|\beta-s|} z^{|\gamma-t|}$ otherwise.

Theorem 20. For all integers $a, b, c>1$ such that

$$
a^{-1}<b^{-1}+c^{-1}, \quad b^{-1}<a^{-1}+c^{-1}, \quad c^{-1}<a^{-1}+b^{-1},
$$

$S(M(a, b, c))$ is finitely generated.

Proof. We show that with respect to our previously defined ordering, $x^{i} y^{j} z^{k}$ can be rewritten as linear combinations of lesser terms whenever $i \geq a, j \geq b$, or $k \geq c$. We accomplish this by choosing a Type I or Type II relation in which 
$x^{i} y^{j} z^{k}$ appears as the greatest term on the left side, as in the previous discussion. We then show that $x^{i} y^{j} z^{k}$ is greater than the greatest term on the right side of the relation. Hence, by subtracting all of the terms less than $x^{i} y^{j} z^{k}$ from both sides of the equation and dividing both sides by the (invertible, as previously discussed) coefficient of $x^{i} y^{j} z^{k}$, we successfully rewrite $x^{i} y^{j} z^{k}$.

Case 1. Suppose $i \geq a$. Let $r=i, \quad s=j$, and $t=k$. Since $r>0$ and $s \geq$ 0 , the greatest term on the left of the Type I relation is $x^{i} y^{j} z^{k}$. Since $a-r=$ $a-i \leq 0$, the greatest term on the right side is $x^{i-a} y^{j-b} z^{k}$ if $j \geq b$ or $i=a$, and $x^{i-a-1} y^{b-j-1} z^{k+1}$ if $j<b$ and $i>a$.

Case 1.1. Suppose $j \geq b$ or $i=a$. Then

$$
\frac{i}{a}+\frac{j}{b}+\frac{k}{c}>\frac{i-a}{a}+\frac{j-b}{b}+\frac{k}{c}
$$

and thus $x^{i} y^{j} z^{k}>x^{i-a} y^{j-b} z^{k}$.

Case 1.2. Suppose $j<b$ and $i>a$. Then

$$
\begin{aligned}
\frac{i}{a}+\frac{j}{b}+\frac{k}{c}>\frac{i}{a}-\frac{j}{b}+\frac{k}{c} & =\frac{i-a}{a}+\frac{b-j}{b}+\frac{k}{c} \\
& >\left(\frac{i-a}{a}+\frac{b-j}{b}+\frac{k}{c}\right)+\left(-\frac{1}{a}-\frac{1}{b}+\frac{1}{c}\right) \\
& =\frac{i-a-1}{a}+\frac{b-j-1}{b}+\frac{k+1}{c} .
\end{aligned}
$$

Hence, $x^{i} y^{j} z^{k}>x^{i-a-1} y^{b-j-1} z^{k+1}$.

Case 2. Suppose $i<a$ and $j \geq b$. Let $r=i, s=j$, and $t=k$. Since $r \geq 0$ and $s>0$, the greatest term on the left of the Type I relation is $x^{i} y^{j} z^{k}$. Since $a-r=a-i>0$ and $b-s=b-j \leq 0$, the greatest term on the right side is $x^{a-i-1} y^{j-b-1} z^{k+1}$ if $j>b$, and $x^{a-i} z^{k}$ if $j=b$.

Case 2.1. Suppose $j>b$. Then

$$
\begin{aligned}
\frac{i}{a}+\frac{j}{b}+\frac{k}{c}>-\frac{i}{a}+\frac{j}{b}+\frac{k}{c} & =\frac{a-i}{a}+\frac{j-b}{b}+\frac{k}{c} \\
& >\left(\frac{a-i}{a}+\frac{j-b}{b}+\frac{k}{c}\right)+\left(-\frac{1}{a}-\frac{1}{b}+\frac{1}{c}\right) \\
& =\frac{a-i-1}{a}+\frac{j-b-1}{b}+\frac{k+1}{c},
\end{aligned}
$$

and thus $x^{i} y^{j} z^{k}>x^{a-i-1} y^{j-b-1} z^{k+1}$. 
Case 2.2. Suppose $j=b$. Then

$$
\frac{i}{a}+\frac{j}{b}+\frac{k}{c}=\frac{i}{a}+1+\frac{k}{c}>-\frac{i}{a}+1+\frac{k}{c}=\frac{a-i}{a}+\frac{k}{c},
$$

and hence $x^{i} y^{j} z^{k}>x^{a-i} z^{k}$.

Case 3. Suppose $i<a, j<b$, and $k \geq c$. Let $r=i, s=j$, and $t=k$. Since $s \geq 0$ and $t>0$, the greatest term on the left of the Type II relation is $x^{i} y^{j} z^{k}$. Since $c-t=c-k \leq 0$, the greatest term on the right side is $x^{i+1} y^{b-j-1} z^{k-c-1}$ if $k>c$, and $x^{i} y^{b-j}$ if $k=c$.

Case 3.1. Suppose $k>c$. Then

$$
\begin{aligned}
\frac{i}{a}+\frac{j}{b}+\frac{k}{c}>\frac{i}{a}-\frac{j}{b}+\frac{k}{c} & =\frac{i}{a}+\frac{b-j}{b}+\frac{k-c}{c} \\
& >\left(\frac{i}{a}+\frac{b-j}{b}+\frac{k-c}{c}\right)+\left(\frac{1}{a}-\frac{1}{b}-\frac{1}{c}\right) \\
& =\frac{i+1}{a}+\frac{b-j-1}{b}+\frac{k-c-1}{c},
\end{aligned}
$$

and thus $x^{i} y^{j} z^{k}>x^{i+1} y^{b-j-1} z^{k-c-1}$.

Case 3.2. Suppose $k=c$. Then

$$
\frac{i}{a}+\frac{j}{b}+\frac{k}{c}=\frac{i}{a}+\frac{j}{b}+1>\frac{i}{a}-\frac{j}{b}+1=\frac{i}{a}+\frac{b-j}{b},
$$

and so $x^{i} y^{j} z^{k}>x^{i} y^{b-j}$.

Remark 21. Note that we can refine the generating set obtained in the proof above, through additional applications of the Type I and Type II relations. For instance, we can rewrite $x^{i} y^{j} z^{k}$ when

- $i<a, j<b$, and $i / a+j / b>1$;

- $i<a, j<b, i / a+j / b=1$, and $i>a / 2$;

- $j<b, k<c$, and $j / b+k / c>1$; or

- $j<b, k<c, j / b+k / c=1$, and $k>c / 2$.

Theorem 22. For all integers $a, b, c>1$ such that

$$
a^{-1}<b^{-1}+c^{-1}, \quad b^{-1}<a^{-1}+c^{-1}, \quad c^{-1}<a^{-1}+b^{-1},
$$

$S(M(a,-b, c))$ is finitely generated.

Proof. We show that with respect to our previously defined ordering, $x^{i} y^{j} z^{k}$ can be rewritten as linear combinations of lesser terms whenever $i \geq a, j \geq b$, or $k>c(2-2 / b)$. As in the previous proof, we accomplish this by choosing a Type I or Type II relation in which $x^{i} y^{j} z^{k}$ appears as the greatest term on the left side, 
and then show that $x^{i} y^{j} z^{k}$ is greater than the greatest term on the right side of the relation. Here, however, the task is a bit more difficult, since the difference in signs prevents us from proceeding in a completely straightforward manner.

Case 1. Suppose $i \geq a$. Let $r=i, s=j$, and $t=k$. Since $r>0$ and $s \geq 0$, the greatest term on the left of the Type I relation is $x^{i} y^{j} z^{k}$. Then $a-r=a-i \leq 0$ and $-b-s=-b-j<0$, and thus $x^{i-a} y^{b+j} z^{k}$ is the greatest term on the right. Then

$$
\frac{i}{a}+\frac{j}{b}+\frac{k}{c}=\frac{i-a}{a}+\frac{b+j}{b}+\frac{k}{c} \quad \text { and } \quad i(k+1)>(i-a)(k+1),
$$

so $x^{i} y^{j} z^{k}>x^{i-a} y^{b+j} z^{k}$.

Case 2. Suppose $i<a$ and $j \geq b$.

Case 2.1. Suppose $k>0$. Let $r=i+1, s=-j-1$, and $t=k-1$. Since $r>0$ and $s<0$, the greatest term on the left of the Type I is relation $x^{i} y^{j} z^{k}=$ $x^{(i+1)-1} y^{-(-j-1)-1} z^{(k-1)+1}$. Since $a-r=a-i-1 \geq 0$ and $-b-s=-b+j+1>0$, $x^{a-i-1} y^{-b+j+1} z^{k-1}$ is the greatest term on the right. Then

$\frac{i}{a}+\frac{j}{b}+\frac{k}{c}>\left(\frac{i}{a}+\frac{j}{b}+\frac{k}{c}\right)+\left(-\frac{1}{a}+\frac{1}{b}-\frac{1}{c}\right)=\frac{a-i-1}{a}+\frac{-b+j+1}{b}+\frac{k-1}{c}$, and thus $x^{i} y^{j} z^{k}>x^{a-i-1} y^{-b+j+1} z^{k-1}$.

Case 2.2. Suppose $k=0$.

Case 2.2.1. Suppose $i>0$. Let $r=i-1, s=-j-1$, and $t=1$. Since $s<0$ and $t>0$, the greatest term on the left of the Type II relation is $x^{i} y^{j}$. Then $-b-s=$ $-b+j+1>0$ and $c-t=c-1>0$, and thus $x^{i-1} y^{-b+j+1} z^{c-1}$ is the greatest term on the right. Then

$$
\frac{i}{a}+\frac{j}{b}+\frac{k}{c}>\left(\frac{i}{a}+\frac{j}{b}+\frac{k}{c}\right)+\left(-\frac{1}{a}+\frac{1}{b}-\frac{1}{c}\right)=\frac{i-1}{a}+\frac{-b+j+1}{b}+\frac{c-1}{c},
$$

and thus $x^{i} y^{j}>x^{i-1} y^{-b+j+1} z^{c-1}$.

Case 2.2.2. Suppose $i=0$. Let $r=0, s=-j$, and $t=0$. Since $t=0$, the greatest term on the left of the Type II relation is $y^{j}$. Then

$$
-b-s=-b+j \geq 0 \text { and } c-t=c>0,
$$

and thus $y^{-b+j} z^{c}$ is the greatest term on the right. Then $j / b=(-b+j) / b+c / c$ and $0(0+1)=0(c+1)$. When $j>b$, we have $\max (j / b, 0)>\max (-b+j / b, c / c)$, and when $j=b$, we have $\max (j / b, 0)=1=\max ((-b+j) / b, c / c)$ and also $j=b>0=-b+j$. Hence $y^{j}>y^{-b+j} z^{c}$.

Case 3. Suppose $i<a, j<b$, and $k>c(2-2 / b)$. (Hence, $k>c$.) 
Case 3.1. Suppose $i>0$. Let $r=i-1, s=-j-1$, and $t=k+1$. Since $s<0$ and $t>0$, the greatest term on the left of the Type II relation is $x^{i} y^{j} z^{k}$. Since $-b-s=-b+j+1 \leq 0$ and $c-t=c-k-1<0$, the greatest term on the right is $x^{i-1} y^{b-j-1} z^{k-c+1}$. Then

$$
\frac{i}{a}+\frac{j}{b}+\frac{k}{c}>\left(\frac{i}{a}+\frac{j}{b}+\frac{k}{c}\right)+\left(-\frac{1}{a}-\frac{1}{b}+\frac{1}{c}\right)=\frac{i-1}{a}+\frac{b-j-1}{b}+\frac{k-c+1}{c},
$$

and thus $x^{i} y^{j}>x^{i-1} y^{b-j-1} z^{k-c+1}$.

Case 3.2. Suppose $i=0$.

Case 3.2.1. Suppose $j=b-1$. Let $r=1, s=-b$, and $t=k-1$. Since $r>0$ and $s<0$, the greatest term on the left of the Type I relation is $y^{b-1} z^{k}$. Then $a-r=a-1>0$ and $-b-s=0$, and thus $x^{a-1} z^{k-1}$ is the greatest term on the right. Since

$$
\frac{b-1}{b}+\frac{k}{c}>\left(\frac{b-1}{b}+\frac{k}{c}\right)+\left(-\frac{1}{a}+\frac{1}{b}-\frac{1}{c}\right)=\frac{a-1}{a}+\frac{k-1}{c},
$$

$y^{b-1} z^{k}>x^{a-1} z^{k-1}$.

Case 3.2.2. Suppose $j<b-1$. Let $r=0, s=j$, and $t=k$. Since $s \geq 0$ and $t>0$, the greatest term on the left of the Type II relation is $y^{j} z^{k} .-b-s=-b-j<0$ and $c-k<0$, and thus, $y^{b+j} z^{k-c}$ is the greatest term on the right. Then

$$
\frac{j}{b}+\frac{k}{c}=\frac{b+j}{b}+\frac{k-c}{c}, \quad 0(k+1)=0(k-c+1),
$$

and $\max (j / b, k / c)=k / c>\max (b+j /(b),(k-c) / c)$ since $k>c((2 b-2) / b) \geq$ $c((b+j) / b)$. Hence $y^{j} z^{k}>y^{b+j} z^{k-c}$.

Proof of Theorem 4. If $\alpha, \beta$ and $\gamma$ are all positive, the result follows by Theorem 20 . If $\alpha, \beta$ and $\gamma$ are all negative, the result follows as well, since $S(M(\alpha, \beta, \gamma))$ is isomorphic to $S(M(-\alpha,-\beta,-\gamma))$.

Suppose that exactly one of $\alpha, \beta$ and $\gamma$ is negative. If $\beta<0$, the result follows by Theorem 22. If $\alpha<0$, by sliding the right handle over the left and performing isotopy, we see that $M(\alpha, \beta, \gamma)$ is identical to $M(\gamma, \alpha, \beta)$, and so the result follows. Similarly, if $\gamma<0$, by sliding the left handle over the right, $M(\alpha, \beta, \gamma)$ is seen to be identical to $M(\beta, \gamma, \alpha)$, and so again the result follows.

If exactly one of $\alpha, \beta$, and $\gamma$ is positive, then $S(M(-\alpha,-\beta,-\gamma))$ is finitely generated, and thus $S(M(\alpha, \beta, \gamma))$ is finitely generated as well.

\section{Examples}

While the previous proofs yield a finite set of generators for $S(M(\alpha, \beta, \gamma))$, they do not exploit the full potential of the Type I and Type II relations. Using the following Python code, we can refine our results for $S(M(a,-b, c))$. 
def greaterthan $(a, b, c, i, j, k, m, n, p)$ :

if $i * b * c+j * a * c+k * a * b>m * b * c+n * a * c+p * a * b:$

return True

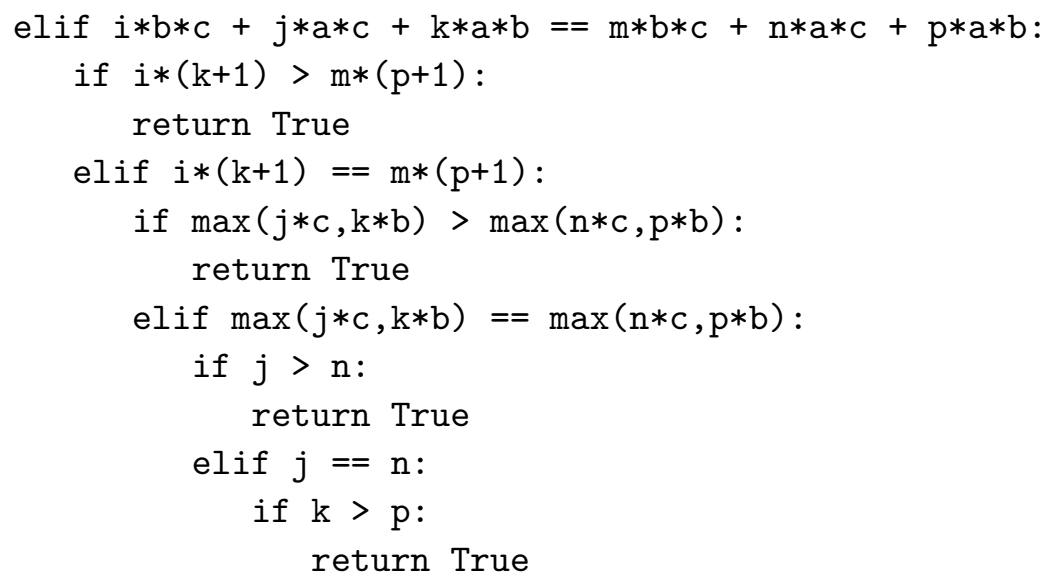

return False

$\operatorname{def} \operatorname{left} 1(i, j, k)$ :

$L=[]$

if $i>0$ or $j>0$ :

$L$. append $([i, j, k])$

$L$. append $([-i,-j, k])$

if $\mathrm{k}>0$ :

$L$. append ( $[i+1,-j-1, k-1])$

$L$. append $([-i-1, j+1, k-1])$

return $\mathrm{L}$

$\operatorname{def} \operatorname{left2}(i, j, k)$ :

$L=[]$

if $\mathrm{j}>0$ or $\mathrm{k}>0$ :

L . append ( $[i, j, k]$ )

$L$. append $([i,-j,-k])$

if $i>0$ :

L . append ( $[i-1, j+1,-k-1])$

L . append ( $[i-1,-j-1, k+1])$

return $\mathrm{L}$

def $\operatorname{right1}(a, b, c, r, s, t)$ :

if $(a-r>0$ and $-b-s<0)$ or $(a-r<0$ and $-b-s>0)$ : return $[a b s(a-r)-1, a b s(-b-s)-1, t+1]$

return $[\mathrm{abs}(\mathrm{a}-\mathrm{r}), \mathrm{abs}(-\mathrm{b}-\mathrm{s}), \mathrm{t}]$ 


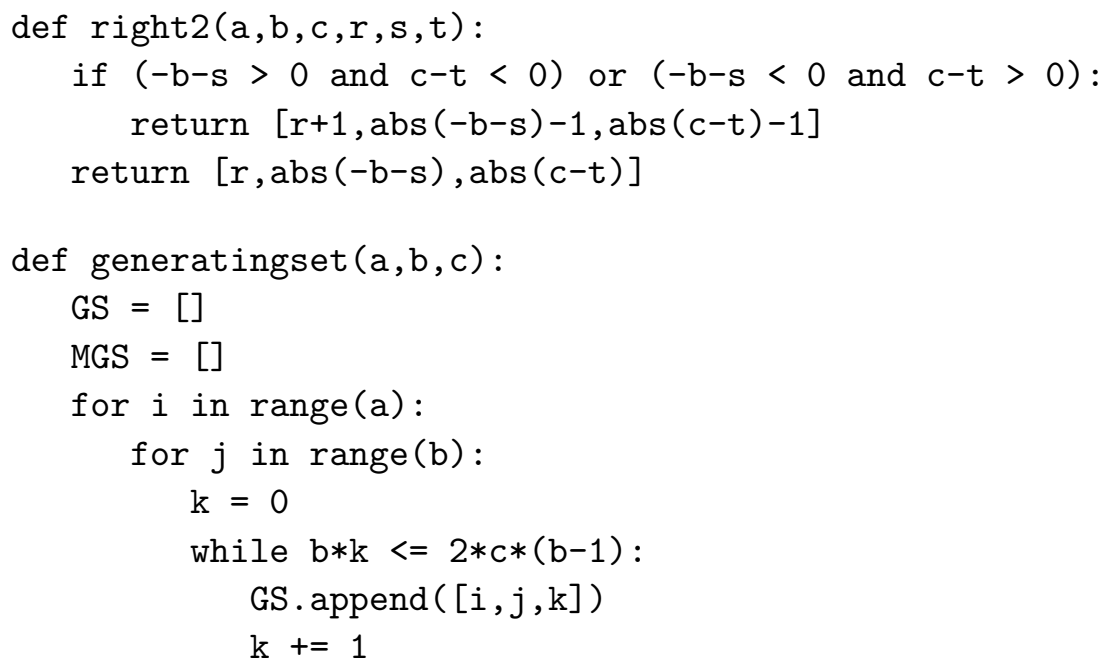

for $\mathrm{T}$ in GS:

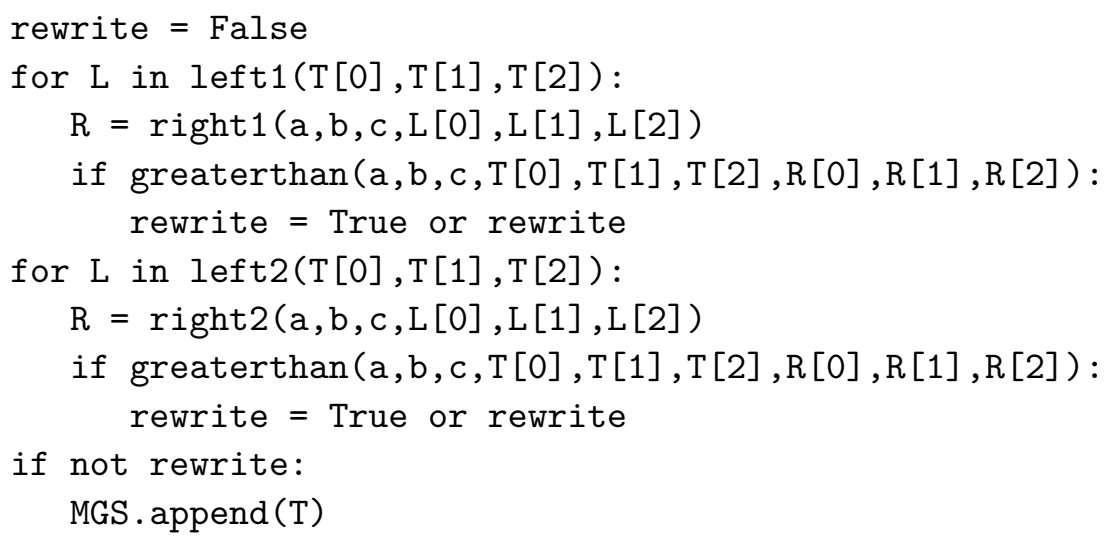

Using this code, we obtain the generating sets listed in the introduction for $S(M(2,-2,2)), S(M(3,-2,3))$, and $S(M(3,-2,5))$, and we find that our generating set is minimal for $S\left(M(2,-2,2) ; \mathbb{Z}\left[A^{ \pm 1}\right], A\right)$.

As for getting minimality of our generating sets for $S\left(M(3,-2,3) ; R\left[A^{ \pm 1}\right], A\right)$ and $S\left(M(3,-2,5) ; R\left[A^{ \pm 1}\right], A\right)$, we might consider $S(M(3,-2,3) ; R,-1)$ and $S(M(3,-2,5) ; R,-1)$, as they are isomorphic to the skein algebras of their fundamental groups, which are generated by representatives of conjugacy classes. For $S\left(M(3,-2,3) ; R\left[A^{ \pm 1}\right], A\right)$, however, this will not help, as only three of the conjugacy classes of the binary tetrahedral group are self-inversive, and hence $S(M(3,-2,3) ; R,-1)$ can be generated by five elements. See [Przytycki and Sikora 2000].

Still, for $S\left(M(3,-2,5) ; R\left[A^{ \pm 1}\right], A\right)$, we can hope to gain some insight, as its conjugacy classes are self-inversive, and since we have the following result: 
Proposition 23. Suppose that a set $L=\left\{L_{1}, \ldots, L_{n}\right\}$ of links in $M$ represents a generating set for $S\left(M ; R\left[A^{ \pm 1}\right], A\right)$.

(1) If $L$ yields a minimal generating set for $S(M ; R,-1)$, then $L$ represents a minimal generating set for $S\left(M ; R\left[A^{ \pm 1}\right], A\right)$.

(2) If $L$ yields a linearly independent set for $S(M ; R,-1)$ and $S\left(M ; R\left[A^{ \pm 1}\right], A\right)$ has no $(A+1)$ torsion, then $L$ represents a basis for $S\left(M ; R\left[A^{ \pm 1}\right], A\right)$.

(3) If $L$ yields a linearly independent set for $S(M ; R,-1)$ and $S\left(M ; R\left[A^{ \pm 1}\right], A\right)$ has torsion, then $S\left(M ; R\left[A^{ \pm 1}\right], A\right)$ has $(A+1)$ torsion.

Proof. (1) Suppose that $L_{n}=f_{1}(A) L_{1}+\cdots+f_{n-1}(A) L_{n-1}$ in $S\left(M ; R\left[A^{ \pm 1}\right], A\right)$. Then $L_{n}=f_{1}(-1) L_{1}+\cdots+f_{n-1}(-1) L_{n-1}$ in $S(M ; R,-1)$, a contradiction.

(2) Suppose that $f_{1}(A) L_{1}+\cdots+f_{n}(A) L_{n}=0$ in $S\left(M ; R\left[A^{ \pm 1}\right], A\right)$. Then in $S(M ; R,-1)$, we have $f_{1}(-1) L_{1}+\cdots+f_{n}(-1) L_{n}=0$. Now $L_{1}, \ldots, L_{n}$ is a basis of $S(M ; R,-1)$, so $f_{i}(-1)=0$ for each $i$, and thus $(A+1) \mid f_{i}$ for each $i$. Hence, $(A+1)\left(g_{1}(A) L_{1}+\cdots+g_{n}(A) L_{n}\right)=0$ for some $g_{1}, \ldots g_{n} . S\left(M ; R\left[A^{ \pm 1}\right], A\right)$ has no $(A+1)$ torsion, so $g_{1}(A) L_{1}+\cdots+g_{n}(A) L_{n}=0$. Hence, $S\left(M ; R\left[A^{ \pm 1}\right], A\right)$ is free.

(3) If $L$ yields a linearly independent set for $S(M ; R,-1)$, and $S\left(M ; R\left[A^{ \pm 1}\right], A\right)$ has torsion, then $L$ cannot represent a basis; and hence $S\left(M ; R\left[A^{ \pm 1}\right], A\right)$ must have $(A+1)$ torsion by $(2)$.

Remark 24. The existence of torsion is a topic of particular interest in skein theory. For example, see the study of $(A+1)$ torsion in [McLendon 2006].

Let $G$ be the binary icosahedral group, with presentation $\left\langle r, s \mid r^{5}=s^{3}=(r s)^{2}\right\rangle$. Since $G$ is finite, the skein algebra of $G$ over $\mathbb{C}$ is isomorphic to $\mathbb{C}[X(G)]$, the $\operatorname{SL}(2, \mathbb{C})$ character variety of $G$, a result of [Przytycki and Sikora 2000]; see also [Bullock 1997b].

Let $\sigma_{0}$ be the trivial 2-dimensional representation of $G$, let $\sigma_{1}$ be the representation of $G$ that sends $r$ and $s$ to

$$
A_{1}=\frac{1}{5}\left[\begin{array}{cc}
-3 e_{5}-e_{5}^{2}+e_{5}^{3}-2 e_{5}^{4} & e_{5}-3 e_{5}^{2}-2 e_{5}^{3}-e_{5}^{4} \\
e_{5}+2 e_{5}^{2}+3 e_{5}^{3}-e_{5}^{4} & -2 e_{5}+e_{5}^{2}-e_{5}^{3}-3 e_{5}^{4}
\end{array}\right]
$$

and

$$
B_{1}=\frac{1}{5}\left[\begin{array}{cc}
-e_{5}-2 e_{5}^{2}-3 e_{5}^{3}-4 e_{5}^{4} & 2 e_{5}-e_{5}^{2}+e_{5}^{3}-2 e_{5}^{4} \\
2 e_{5}-e_{5}^{2}+e_{5}^{3}-2 e_{5}^{4} & -4 e_{5}-3 e_{5}^{2}-2 e_{5}^{3}-e_{5}^{4}
\end{array}\right],
$$

respectively, and let $\sigma_{2}$ be the representation of $G$ that sends $r$ and $s$ to

$$
A_{2}=\left[\begin{array}{cc}
e_{5}-e_{5}^{2} & -e_{5}^{2}-e_{5}^{4} \\
-e_{5}-e_{5}^{4} & -e_{5}-e_{5}^{3}
\end{array}\right] \text { and } B_{2}=\left[\begin{array}{cc}
1 & -e_{5}^{3} \\
e_{5}^{2} & 0
\end{array}\right],
$$

respectively, where $e_{5}=e^{2 \pi i / 5}$. 
Using GAP [GAP 2007], we can see that $\sigma_{0}, \sigma_{1}$, and $\sigma_{2}$ are $\operatorname{SL}(2, \mathbb{C})$ representations of $G$, and any $\operatorname{SL}(2, \mathbb{C})$ representation $\sigma$ of $G$ is equivalent to one of them: If irreducible, $\sigma$ is equivalent to $\sigma_{1}$ or $\sigma_{2}$, and if reducible, $\sigma$ is equivalent to $\sigma_{0}$, since $G$ is perfect. See [Culler and Shalen 1983].

Let $\chi_{0}, \chi_{1}$, and $\chi_{2}$ be the characters of $\sigma_{0}, \sigma_{1}$, and $\sigma_{2}$, respectively, and for each $g \in G$, let $\tau_{g}$ be the evaluation map defined on the characters of $G$ by $\tau_{g}(\chi)=\chi(g)$. Note that since $1, r, r^{2}, r^{3}, r^{4}, r^{5}, r s, s$, and $s^{2}$ represent the conjugacy classes of $G, \mathbb{C}[X(G)]$ is generated by $\tau_{1}, \tau_{r}, \tau_{r^{2}}, \tau_{r^{3}}, \tau_{r^{4}}, \tau_{r^{5}}, \tau_{r s}, \tau_{s}$, and $\tau_{s^{2}}$.

\begin{tabular}{c|ccccccccc} 
& $\tau_{1}$ & $\tau_{r}$ & $\tau_{r^{2}}$ & $\tau_{r^{3}}$ & $\tau_{r^{4}}$ & $\tau_{r^{5}}$ & $\tau_{r s}$ & $\tau_{s}$ & $\tau_{s^{2}}$ \\
\hline$\chi_{0}$ & 2 & 2 & 2 & 2 & 2 & 2 & 2 & 2 & 2 \\
$\chi_{1}$ & 2 & $-e_{5}-e_{5}^{4}$ & $e_{5}^{2}+e_{5}^{3}$ & $-e_{5}^{2}-e_{5}^{3}$ & $e_{5}+e_{5}^{4}$ & -2 & 0 & 1 & -1 \\
$\chi_{2}$ & 2 & $-e_{5}^{2}-e_{5}^{3}$ & $e_{5}+e_{5}^{4}$ & $-e_{5}-e_{5}^{4}$ & $e_{5}^{2}+e_{5}^{3}$ & -2 & 0 & 1 & -1
\end{tabular}

From the table, we can see that the following relations hold in $\mathbb{C}[X(G)]$ :

$$
\begin{array}{lll}
\tau_{s^{2}}=3 \tau_{s}-2 \tau_{1}, & \tau_{r s}=2 \tau_{s}-\tau_{1}, & \tau_{r^{5}}=4 \tau_{s}-3 \tau_{1}, \\
\tau_{r^{4}}=4 \tau_{s}-\tau_{r}-2 \tau_{1}, & \tau_{r^{3}}=3 \tau_{s}-\tau_{r}-\tau_{1}, & \tau_{r^{2}}=\tau_{s}+\tau_{r}-\tau_{1}
\end{array}
$$

Furthermore, $\left\{\tau_{1}, \tau_{r}, \tau_{s}\right\}$ are linearly independent in $\mathbb{C}[X(G)]$, since the matrix

$$
\left[\begin{array}{lll}
\tau_{1}\left(\chi_{0}\right) & \tau_{r}\left(\chi_{0}\right) & \tau_{s}\left(\chi_{0}\right) \\
\tau_{1}\left(\chi_{1}\right) & \tau_{r}\left(\chi_{1}\right) & \tau_{s}\left(\chi_{1}\right) \\
\tau_{1}\left(\chi_{2}\right) & \tau_{r}\left(\chi_{2}\right) & \tau_{s}\left(\chi_{2}\right)
\end{array}\right]=\left[\begin{array}{ccc}
2 & 2 & 2 \\
2 & -e_{5}-e_{5}^{4} & 1 \\
2 & -e_{5}^{2}-e_{5}^{3} & 1
\end{array}\right]
$$

is invertible.

Thus, $S(M(3,-2,5) ; \mathbb{C},-1)$ is 3 -dimensional, and therefore we cannot use Proposition 23 to show that our generating set for $S\left(M(3,-2,5) ; \mathbb{C}\left[A^{ \pm 1}\right], A\right)$ is minimal. Hence, we are left with the following:

Question. For some ring $R$ and unit $A$, is $\left\{1, z, z^{2}, z^{3}, z^{4}, z^{5}, y, x, x^{2}\right\}$ a minimal generating set for $S(M(3,-2,5) ; R, A)$ ? If not, it is generated by $\{1, z, x\}$ for every ring $R$ and unit $A$ ?

\section{Acknowledgments}

I would like to thank Patrick Gilmer for his suggestions and encouragement.

\section{References}

[Bullock 1997a] D. Bullock, "On the Kauffman bracket skein module of surgery on a trefoil", Pacific J. Math. 178:1 (1997), 37-51. MR 98i:57006 Zbl 0878.57005

[Bullock 1997b] D. Bullock, "Rings of $\mathrm{SL}_{2}(\mathbb{C})$-characters and the Kauffman bracket skein module", Comment. Math. Helv. 72:4 (1997), 521-542. MR 98k:57008 Zbl 0907.57010 
[Culler and Shalen 1983] M. Culler and P. B. Shalen, "Varieties of group representations and splittings of 3-manifolds", Ann. of Math. (2) 117:1 (1983), 109-146. MR 84k:57005 Zbl 0529.57005

[GAP 2007] The GAP Group, "GAP: Groups, Algorithms, and Programming”, 2007, Available at http://www.gap-system.org/. Version 4.4.10.

[Gilmer and Harris 2007] P. M. Gilmer and J. M. Harris, "On the Kauffman bracket skein module of the quaternionic manifold", J. Knot Theory Ramifications 16:1 (2007), 103-125. MR 2007m:57015 Zbl 1117.57009

[Hoste and Przytycki 1993] J. Hoste and J. H. Przytycki, "The $(2, \infty)$-skein module of lens spaces; a generalization of the Jones polynomial", J. Knot Theory Ramifications 2:3 (1993), 321-333. MR 95b:57010 Zbl 0796.57005

[Hoste and Przytycki 1995] J. Hoste and J. H. Przytycki, "The Kauffman bracket skein module of $S^{1} \times S^{2}$ ", Math. Z. 220:1 (1995), 65-73. MR 96f:57006 Zbl 0826.57007

[Kauffman 1988] L. H. Kauffman, "New invariants in the theory of knots", Amer. Math. Monthly 95:3 (1988), 195-242. MR 89d:57005 Zbl 0657.57001

[McLendon 2006] M. McLendon, "Detecting torsion in skein modules using Hochschild homology”, J. Knot Theory Ramifications 15:2 (2006), 259-277. MR 2007a:57017 Zbl 05017925

[Przytycki 1991] J. H. Przytycki, "Skein modules of 3-manifolds", Bull. Polish Acad. Sci. Math. 39:1-2 (1991), 91-100. MR 94g:57011 Zbl 0762.57013

[Przytycki and Sikora 2000] J. H. Przytycki and A. S. Sikora, "On skein algebras and $\mathrm{Sl}_{2}(\mathbb{C})$ character varieties”, Topology 39:1 (2000), 115-148. MR 2000g:57026 Zbl 0958.57011

[Rolfsen 1976] D. Rolfsen, Knots and links, Mathematics Lecture Series 7, Publish or Perish, Berkeley, CA, 1976. MR 58 \#24236 Zbl 0339.55004

[Turaev 1988] V. G. Turaev, "The Conway and Kauffman modules of a solid torus", Zap. Nauchn. Sem. Leningrad. Otdel. Mat. Inst. Steklov. (LOMI) 167:Issled. Topol. 6 (1988), 79-89, 190. In Russian; translated in J. Soviet Math. 52:1 (1990), 2799-2805. MR 90f:57012 Zbl 0673.57004

Received July 11, 2008. Revised August 1, 2008.

JOHN M. HARRIS

DEPARTMENT OF MATHEMATICS

UNIVERSITY OF SOUTHERN MISSISSIPPI GULF COAST

730 EAST BEACH BOULEVARD 5128

LONG BEACH, MS 39560

UNITED STATES

john.m.harris@usm.edu

http://ocean.otr.usm.edu/ w487892/ 